\title{
Les producteurs d'ovins et les éleveurs laitiers extensifs sont les principaux bénéficiaires de l'application française du bilan de santé de la PAC
}

\author{
V. CHATELLIER I, J.-C. GUESDON², H. GUYOMARD ${ }^{3}$, C. PERROT ${ }^{2}$ \\ ${ }^{1}$ INRA, UR1134 LERECO, F-44316 Nantes, France \\ 2 Institut de l'Elevage, GEB, 149 Rue de Bercy, F-75012 Paris, France \\ ${ }^{3}$ INRA, Collège de Direction, 147 rue de l'Université, F-75338 Paris, France
}

Courriel :vchatel@nantes.inra.fr

Les mesures adoptées en France dans le cadre du bilan de santé de la PAC auront, à compter de l'année 2010, un impact économique positif pour les exploitations spécialisées en production ovine et en production laitière extensive. Cette réorientation des aides directes entre catégories d'exploitations ne doit cependant pas conduire à occulter les débats engagés sur la justification, à plus long terme, des soutiens octroyés à l'agriculture.

Les exploitations productrices de viande bovine et de viande ovine bénéficiaient, déjà avant la réforme de la Politique Agricole Commune (PAC) de 1992, d'aides directes allouées au travers des primes animales ou des Indemnités Compensatoires de Handicaps Naturels (ICHN). A partir de 1992, date à laquelle une baisse importante du prix institutionnel des céréales a été engagée, les exploitations céréalières sont devenues, elles aussi, progressivement de plus en plus dépendantes des aides directes (lesquelles étaient appelées à l'époque paiements compensatoires). Dans le secteur laitier, il a fallu attendre la réforme de l'Organisation Commune de Marché (OCM) du lait et des produits laitiers de 2003 pour que les exploitations de ce secteur deviennent, elles aussi, pleinement concernées, même si elles percevaient déjà des aides directes au titre de leurs productions agricoles associées (céréales, viande bovine...). La montée en puissance des aides directes à l'agriculture suscite légitimement de nombreux débats au sein des pouvoirs publics, des organisations professionnelles agricoles, de la sphère des économistes (Guyomard et al 2007, Bureau et Mahé 2008, Bazin 2010, Boussard et Trouvé 2010, Bureau et Witzke 2010) ou, plus globalement, de la société. Ces débats s'inscrivent aujourd'hui dans un contexte difficile marqué notamment par les négociations engagées sur les perspectives financières de l'Union Européenne pour la période 2014-2020, le retard dans l'avancement du cycle de Doha relativement aux négociations commerciales multilatérales et la forte volatilité des prix agricoles.

Les précédentes réformes de la PAC (1992, 1999 et 2003) se sont toutes orientées dans le sens d'une baisse des prix garantis compensée, partiellement, par l'octroi de paiements directs aux agriculteurs (Butault et al 2005). A l'origine, ces aides directes ont été déterminées, dans chaque exploitation, sur la base des facteurs de production (terre et cheptel). Ainsi, le montant des aides directes par exploitation était, pour un secteur productif donné, assez directement relié à la taille de la structure. Ces réformes de la PAC ont également conduit, grâce au processus dit de la modulation des aides directes du premier pilier de la PAC, à une augmentation des fonds alloués en faveur du deuxième pilier, à savoir le développement rural (notamment des mesures agri-environnementales). La réforme de 2003 a donné lieu à deux innovations majeures (Borzeix et al 2006, Bureau 2007). La première concerne le renforcement du principe de la conditionnalité des aides directes. Cela signifie que le versement des soutiens directs aux agriculteurs est désormais subordonné au respect d'une part de normes européennes relatives à l'environnement, la santé des plantes et des animaux et le bien-être animal, et d'autre part à des règles nationales relatives aux Bonnes Conditions Agricoles et Environnementales (BCAE). La seconde porte sur l'instauration d'un découplage des mesures de soutien des revenus agricoles (OCDE 2001). Le Conseil européen a fait ce choix sous la pression des mesures arrêtées dans le cadre de l'Accord Agricole du Cycle de l'Uruguay (AACU), plus précisément de façon à ce que les aides directes de la PAC puissent être intégrées dans la «boîte verte» qui regroupe les soutiens ayant sur la production et les échanges un effet de distorsion nul ou au plus minime mais aussi afin de mieux maîtriser les dépenses budgétaires pour l'agriculture lors des élargissements ultérieurs (2004 et 2007). Le découplage s'est traduit par l'instauration des Droits à Paiement Unique (DPU) qui ont été déterminés, du moins en France, sur la base des montants historiques d'aides directes couplées. Dans le cadre du bilan de santé de la PAC arrêté le 20 novembre 2008, les autorités communautaires ont, de nouveau, modifié certaines règles, pour une application prévue entre 2010 et 2015 (selon les mesures). Ainsi, les Etats membres ont l'obligation de recourir à un découplage total des soutiens directs et non plus seulement à un découplage partiel (comme le permettait le règlement de 2003) à l'exception de la prime à la 
vache allaitante ainsi que des primes aux ovins et aux caprins; les Etats membres sont invités à rendre le montant des DPU par hectare plus homogène entre exploitations agricoles; le taux de modulation des aides directes du premier pilier est augmenté (de 5 à $10 \%)$; l'obligation de mettre en jachère une partie de la sole est supprimée; enfin, le régime des quotas laitiers sera abandonné à horizon 2015 .

Les décisions communautaires du bilan de santé de la PAC laissent cependant d'importantes marges de manœuvre aux Etats membres, singulièrement sur la manière de répartir les aides directes entre catégories d'agriculteurs. $\mathrm{Si}$ ces latitudes offrent aux différents gouvernements la possibilité d'orienter la politique agricole, elles conduisent aussi à une complexification croissance de la PAC à l'échelle communautaire, chaque pays ayant ses propres modalités d'application des règlements européens. En France, le Ministre en charge de l'agriculture (Michel Barnier), qui présidait le Conseil des ministres au moment de l'adoption de la réforme de 2008, a décidé de se saisir des opportunités offertes par ces nouveaux règlements pour réorienter partiellement les aides directes à l'agriculture française. Il n'est pas inutile de rappeler ici que cette décision s'inscrivait dans un contexte où la hausse du prix des céréales rendait à l'époque plus facile une décision de réorientation des soutiens du secteur des grandes cultures vers le secteur de l'élevage. Les décisions nationales relatives à l'application du bilan de santé de la PAC ont donc été arrêtées le 23 février 2009, certaines modalités d'application ayant ensuite été précisées dans le courant de l'été 2009.

Dans ce cadre, l'objet de cet article est de rendre compte des effets supposés de ces décisions sur l'évolution des aides directes (et donc du revenu) de différentes catégories d'exploitations agricoles. Trois parties sont distinguées. La première rappelle les principales décisions prises, tant à l'échelle communautaire que nationale, relativement au bilan de santé de la PAC, en se focalisant uniquement sur la question des soutiens directs. La deuxième développe, de manière synthétique, la méthode et les hypothèses retenues dans les simulations établies à partir du Réseau d'Information Comptable Agricole (RICA). La troisième présente les résultats de ces simulations en cherchant à mettre en évidence les principaux effets différenciés de ces décisions sur les exploitations agricoles selon leur système de production et leur localisation géographique.

\section{1 / Le bilan de santé de la PAC et les soutiens directs}

La présentation des mesures du bilan de santé de la PAC est effectuée en deux étapes : la première précise les principales évolutions inscrites dans les règlements communautaires relativement aux soutiens directs; la seconde présente les arbitrages du Ministre français en charge de l'agriculture.

\section{1 / Le règlement communau- taire et les latitudes offertes aux Etats membres}

Le bilan de santé de la PAC prévoit l'instauration obligatoire à horizon 2012 d'un découplage total de toutes les aides directes du premier pilier de la PAC, à l'exception, pour les pays qui le souhaitent (Gohin 2009), de la Prime au Maintien du Troupeau de Vaches Allaitantes (PMTVA) et de la Prime à la Brebis et à la Chèvre $(\mathrm{PBC})$. Il s'agit là d'une étape supplémentaire importante dans le processus de réforme des modalités d'octroi des soutiens directs. En 2003, les autorités communautaires avaient en effet donné aux Etats membres la possibilité de maintenir couplées certaines primes, essentiellement dans l'optique d'éviter le risque d'un abandon de certaines productions dans les zones défavorisées, notamment celles de montagne (Chatellier et Delattre 2005). Dans ce cadre, et conformément à ce que permettait le règlement communautaire, la France avait opté pour une stratégie qui consistait à maintenir couplées $100 \%$ de la PMTVA, $100 \%$ de la Prime à l'abattage (PAB) des veaux, $40 \%$ de la PAB des gros bovins, $50 \%$ de la Prime à la brebis (PB) et $25 \%$ des aides directes aux superficies de céréales et d'oléoprotéagineux. Dans d'autres Etats membres (Irlande et RoyaumeUni), les gouvernements ont décidé de recourir immédiatement au découplage total. L'Allemagne, l'Italie et la Grèce, ont également retenu le découplage total, à l'exception des aides aux cultures spécifiques. L'Autriche, la Belgique, l'Espagne ou les Pays-Bas ont, comme en France, appliqué un couplage partiel au profit des productions animales (Boinon et al 2008).

Outre le passage obligatoire au découplage total, le bilan de santé de la PAC incite, mais sans le contraindre, les Etats membres à aller dans le sens d'une plus grande homogénéité, entre agriculteurs d'une même région, du montant des DPU alloués par hectare. Cette suggestion vise à répondre à la critique selon laquelle il sera de plus en plus difficile de justifier, au fil du temps, l'octroi de soutiens budgétaires aux exploitations agricoles sur la seule base de leurs orientations productives et de leurs situations structurelles passées. Ainsi, les autorités communautaires souhaitent que les Etats membres (pour ceux qui sont encore concernés, dont la France) s'écartent progressivement du modèle dit «historique» d'allocation des DPU. Dans ce modèle, le montant du paiement unique perçu par exploitation correspond au montant des aides directes (du premier pilier) alloué au cours de la période de référence 2000-2002 (Chatellier 2006, Piet et al 2006). Par construction, ce modèle historique conduit à figer la répartition initiale des soutiens directs entre exploitations, exception faite des effets liés à la restructuration. Dans plusieurs pays, dont l'Allemagne ou le Royaume-Uni, les autorités nationales ont décidé de ne pas retenir le modèle historique. Ils ont préféré une méthode alternative qui permettait, contrairement au modèle historique, de redistribuer une partie des soutiens directs entre exploitations. Sans entrer ici dans une présentation détaillée des modèles retenus, il convient simplement de retenir que ceux-ci entrainaient une uniformisation progressive du montant du DPU alloué par hectare à l'intérieur d'une zone géographique donnée (chaque agriculteur de la zone privilégiée bénéficiant d'un DPU par hectare identique, ce qui n'était pas le cas avant l'application du découplage en 2005).

Le règlement communautaire $\mathrm{n}^{\circ} 73 /$ 2009 du Conseil issu du bilan de santé de la PAC donne aux Etats membres la possibilité d'utiliser une «boîte à outils» pour réorienter une part des soutiens directs à l'agriculture. Ainsi, quatre instruments peuvent être mobilisés :

- le premier (la modulation volontaire) offre la possibilité aux Etats membres de recourir à une modulation complémentaire à la modulation obligatoire ;

- le second (articles 45 à 47 du règlement) autorise un Etat membre ayant recours au modèle historique (comme c'est le cas en France) à procéder à des modifications progressives à la valeur des droits en fonction de critères objectifs et non discriminatoires tels que le potentiel agricole et l'environnement. Les fonds qui en résultent sont ensuite alloués sur une base régionale ;

- le troisième (article 63 du règlement) autorise un Etat membre à utiliser tout ou partie des ressources budgétaires fruit d'un découplage accru pour définir de nouveaux droits à paiement ou augmenter leur valeur sur la base des activités agricoles telles que celles basées sur l'herbe ou exigeant des animaux ; cette réorientation doit se faire selon des critères objectifs et non 
discriminatoires tels que le potentiel agricole ou l'environnement ;

- le quatrième (article 68 du règlement) autorise un pays à prélever jusqu'à $10 \%$ des aides directes du premier pilier pour les réaffecter sur cinq objectifs : l'environnement, la qualité et la commercialisation des produits; la compensation d'handicaps géographiques ou sectoriels; la revalorisation des paiements découplés à l'hectare dans les zones présentant un risque de déprise agricole; la prise en charge d'une partie des primes d'assurance contre les risques dans le secteur des grandes cultures; la participation aux fonds mutualisés de lutte contre les maladies animales et végétales.

\section{2 / Les choix français pour réorienter les soutiens}

Les décisions nationales prises relativement à l'application du bilan de santé de la PAC préservent le modèle «historique» d'attribution des DPU. Ainsi, le Ministre en charge de l'agriculture n'a pas souhaité se saisir des opportunités laissées par l'article 47, option privilégiée dès l'instauration du découplage par l'Allemagne et le Royaume-Uni. Il n'a pas non plus décidé de recourir à la modulation volontaire. Pour autant, et grâce à l'utilisation des deux derniers outils précités (articles 63 et 68 du règlement), il s'est orienté dans le sens d'une certaine redistribution des soutiens entre catégories d'agriculteurs (APCA 2009). Cette dernière visait à conforter les productions agricoles jugées fragiles (notamment la production ovine où les revenus moyens sont historiquement bas et les élevages laitiers de montagne qui seront confrontés aux effets induits de la suppression future des quotas laitiers), à soutenir les superficies en herbe et à instaurer de nouveaux outils de gestion des risques. Selon les propres termes du Ministre, l'objectif affiché est d'orienter la PAC sur une voie «plus juste, plus équitable et plus préventive»" (Ministère de l'Agriculture et de la Pêche 2009).

En cumulant les fonds issus de l'augmentation du taux de la modulation obligatoire (de 5 à $10 \%$ ) et ceux correspondants à l'utilisation des articles 63 et $68 \mathrm{du}$ règlement, le prélèvement budgétaire total (France) opéré sur les aides directes (premier pilier) représente 1,5 milliard d'euros (simulation à horizon 2013). Cela correspond à $12 \%$ des concours publics (communautaires et nationaux) alloués à l'agriculture et aux territoires ruraux en France (12 milliards d'euros en 2009). Ces derniers regroupent notamment 5,7 milliards d'euros d'aides découplées (DPU), 2,5 milliards d'euros d'aides couplées et 1,5 milliard d'euros d'aides du deuxième pilier (CCAN 2010).

Les montants prélevés (1,5 milliard d'euros) sont réaffectés à hauteur de 1,26 milliard d'euros, le solde (240 millions d'euros) permettant d'assurer la pérennisation du financement de la Prime Herbagère AgriEnvironnementale (PHAE). Comme cela est précisé dans le tableau 1, les fonds prélevés au titre de l'article 63 (767 millions d'euros dont 640 millions d'euros d'aides directes aux superficies de céréales et d'oléoprotéagineux, soit $55,5 \%$ des aides encore couplées à cette date, et 127 millions d'euros de primes animales, soit $12,5 \%$ des aides encore couplées à cette date) sont orientés vers les surfaces productives d'herbe (707 millions d'euros), les surfaces de maïs fourrage (30 millions d'euros) et les légumes de plein champ (30 millions d'euros). A l'exception de $75 \%$ de la PMTVA qui restent couplés, les aides végétales $(44,5 \%)$ et animales hors PMTVA $(87,5 \%)$ non prélevées au titre de l'article 63 sont totalement découplées et viennent conforter les DPU historiques de chaque exploitation, ainsi que la moitié des $25 \%$ de PMTVA qui sont découplés.

Les fonds prélevés au titre de l'article 68 (472 millions d'euros) sont orientés vers une série de nouvelles aides ciblées. Celles-ci concernent (tableau 1) notamment le secteur des ovins-caprins (135 millions d'euros), la gestion des risques par l'assurance récolte (100 millions d'euros), l'agriculture biologique (50 millions d'euros), les exploitations laitières de montagne (45 millions d'euros) et les protéines végétales (40 millions d'euros). Les fonds résiduels prélevés, qui correspondent à l'augmentation du taux de modulation, sont utilisés pour honorer des décisions prises précédemment (refinancement de la PHAE et revalorisation de l'ICHN) et pour faire face à des dépenses nouvelles comme la mesure agri-environnementale «rotationnelle». Cette dernière est réservée aux exploitations céréalières (ayant plus de $60 \%$ de céréales et d'oléoprotéagineux dans la surface agricole utilisée) des zones intermédiaires (rendement de référence inférieur à $60 \mathrm{qx}$ par hectare) diversifiant leur assolement (avec un minimum de $5 \%$ d'oléoprotéagineux).

\section{2 / Outil statistique, métho- de de simulation et scéna- rios testés}

Partant de ces décisions nationales de réorientation des aides directes à l'agriculture, l'objectif de cet article est de discuter, au travers de simulations appliquées aux données du RICA, des effets attendus de ces décisions pour les différentes catégories d'exploitations agricoles françaises.

\section{1 / L'outil statistique et la méthode de simulation}

Les simulations sont appliquées aux données individuelles du RICA (exercice 2007), c'est-à-dire à chaque exploitation de l'échantillon (lequel compte près de 8000 exploitations en France). Cela signifie que les résultats moyens présentés pour tel ou tel groupe d'exploitations correspondent à une agrégation des effets individuels. Seul l'outil statistique du RICA permet de procéder à ce type de simulation à une échelle nationale dans la mesure où il regroupe des informations harmonisées et détaillées sur la structure, les résultats économiques et la situation financière des exploitations. Cet outil statistique est construit pour être représentatif des 320000 exploitations agricoles françaises dites professionnelles (au sens des indicateurs de la statistique agricole). Chaque exploitation de l'échantillon est doté d'un coefficient de pondération et est sélectionnée en fonction d'une grille typologique à trois entrées : la région administrative, l'orientation technicoéconomique (OTEX) et la dimension économique (marge brute standard).

Les simulations menées ici visent à mesurer l'impact comptable des décisions prises et présentées précédemment. L'impact est mesuré par rapport à la situation de référence (modèle historique), toutes choses égales par ailleurs, c'est-à-dire sans anticiper une éventuelle adaptation des agriculteurs à la nouvelle donne et sans tenir compte des gains de productivité, lesquels diffèrent selon les catégories d'exploitations agricoles. De même, les évolutions des prix des produits et/ou des facteurs de production ne sont pas prises en compte, que celles-ci soient générées par les décisions ou exogènes à celles-ci.

L'impact est exprimé en euros par exploitation, en pourcentage des aides directes et du résultat courant avant impôt (ou revenu). Pour tenir compte de la forte variabilité du prix des produits agricoles au cours de la période récente (notamment pour les céréales et le lait), l'impact est rapporté à un revenu moyen calculé sur deux années (2006-2007).

\section{2 / Les principales hypothèses retenues pour l'affectation des fonds}

La simulation relative aux prélèvements budgétaires opérés (modulation obligatoire et articles 63 et 68 ) ne pose 
Tableau 1. La réorientation des soutiens budgétaires (estimation annuelle en 2013) induite par les décisions nationales du bilan de santé de la PAC du 23 février 2009.

\begin{tabular}{|c|c|}
\hline Affectation des financements & Source des financements \\
\hline $\begin{array}{l}\text { [l] «Nouveau soutien à l'élevage» : } \\
970 \text { millions d'euros }\end{array}$ & \\
\hline $\begin{array}{l}\text { (1) Aide sur les surfaces d'herbe productives }(700) \\
\left({ }^{*}\right) \text { PHAE }(240 \text {, dont } 64 \text { de contreparties nationales) } \\
\text { (2) Aides aux fourrages (30) } \\
\text { [II] «Consolider les productions fragiles» : } \\
\mathbf{2 6 5} \text { millions d'euros }\end{array}$ & $\begin{array}{l}\text { Article } 63 \\
\text { Modulation additionnelle de } 5 \% \\
\text { Article } 63\end{array}$ \\
\hline $\begin{array}{l}\text { (3) Ovins et caprins }(135) \\
\text { (4) Lait de montagne }(45) \\
\text { (5) Blé dur des zones traditionnelles }(8) \\
\text { (6) Veaux sous la mère }(4,6) \\
\text { (7) Légumes de plein champ et pommes de terres }(30) \\
\text { (8) Revalorisation de l'ICHN (42, dont } 19 \text { de contreparties nationales) } \\
\text { [III] «Soutenir les systèmes de production durables»: } \\
129 \text { millions d'euros }\end{array}$ & $\begin{array}{l}\text { Article } 68 \\
\text { Article } 68 \\
\text { Article } 68 \\
\text { Article } 68 \\
\text { Article } 63 \\
\text { Modulation additionnelle à } 5 \%\end{array}$ \\
\hline $\begin{array}{l}\text { (9) Protéines végétales (40) } \\
\text { (10) AB, maintien et conversion ( } 57 \text {, dont } 3 \text { de contreparties nationales) } \\
\text { (11) Nouveaux défis ( } 32 \text {, dont } 14 \text { de contreparties nationales) } \\
\text { [IV] «Instaurer un dispositif de couverture des risques»: } \\
140 \text { millions d'euros }\end{array}$ & $\begin{array}{l}\text { Article } 68 \\
\text { Article } 68 \text { (et modulation additionnelle) } \\
\text { Modulation additionnelle à } 5 \%\end{array}$ \\
\hline $\begin{array}{l}\text { (12) Assurance récolte (100, hors budget du MAP et contribution des professionnels) } \\
\text { (13) Fonds sanitaire ( } 40 \text {, hors budget du MAP et contribution des professionnels) } \\
\text { Total [I] à [IV] : } 1504 \text { millions d'euros (dont } 100 \text { de contreparties nationales) } \\
\text { Moins PHAE : } 240 \text { millions d'euros (dont } 64 \text { de contreparties nationales) }\end{array}$ & $\begin{array}{l}\text { Article } 68 \\
\text { Article } 68\end{array}$ \\
\hline Total redéployé : 1264 millions d'euros (dont 36 de contreparties nationales) & Mobilisation de fonds non utilisés \\
\hline $\begin{array}{l}\text { Plan complémentaire du } 30 \text { mars } 2009 \\
-\quad 90 \text { millions d'aide à la diversité des assolements (pour } 2010 \text { uniquement, } \\
\text { non retenu dans les simulations effectuées à réforme accomplie) } \\
\text { - } \quad 60 \text { millions pour la mesure agroenvironnementale rotationnelle }\end{array}$ & Modulation additionnelle à $5 \%$ \\
\hline
\end{tabular}

pas de difficultés particulières dans la mesure où les variables renseignées dans le RICA sont suffisamment précises pour que la simulation soit robuste. Celle relative à l'affectation des fonds dans chaque exploitation est, en revanche, moins aisée et doit faire l'objet d'hypothèses plus ou moins discutables. Partant des treize postes mentionnés dans le tableau 1, il s'agit en effet de choisir une voie qui soit la moins imparfaite possible pour procéder à l'affectation des fonds. En l'occurrence, les hypothèses formulées n'engagent que notre responsabilité et non celle des pouvoirs publics en charge du dossier. Une des difficultés de l'exercice tient au fait que le montant unitaire de certaines primes (à l'hectare ou à la tête de bétail) ne sera calculé que in fine, une fois que le nombre total de demandes sera connue (ce pour respecter le plafond de l'enveloppe budgétaire). Dans la simulation, trois nouveaux fonds ne sont cependant pas pris en compte. Il s'agit des 100 millions d'euros prévus au titre de l'assurance récolte, notamment sur les productions agricoles les plus exposées aux risques (arboriculture, viticulture); des 40 millions d'euros prévus au titre du fonds sanitaire (pour indemniser les incidents sanitaires survenus sur les productions animales et végétales); des 32 millions d'euros prévus au titre des «nouveaux défis» (amélioration de la performance énergétique des entreprises agricoles, gestion de l'eau et la biodiversité).

- Pour les 707 millions d'euros de soutiens directs alloués aux superficies de prairies, les choix opérés dans la simulation tiennent compte des arbitrages rendus en début d'été 2009. Les superficies éligibles à cette prime recouvrent les prairies permanentes et les prairies temporaires considérées comme «productives». Toutes les surfaces de prairies productives ne sont cependant pas identiques face à cette nouvelle aide. Plus précisément, les superficies sont comptabilisées de manière distincte en fonction du taux de chargement de l'exploitation (exprimé en UGB herbivores par hectare de Surface Fourragère Principale (SFP), les surfaces de parcours étant prises en compte dans les surfaces fourragères) et de l'importance des surfaces de prairies dans l'exploitation (avec un seuil fixé à 50 ha par exploitation ou associé de GAEC). Le dispositif est aussi ouvert aux exploitations dont le chargement est inférieur à $0,5 \mathrm{UGB} / \mathrm{ha}$, avec un plafonnement des surfaces pour ramener le taux de chargement à $0,5 \mathrm{UGB} /$ ha et un plafond de 50 ha pour bénéficier de l'aide. Autrement dit, pour ces exploitations, le soutien maximum sera de 2500 euros par exploitation. 
- Les 125 millions d'euros d'aides attribués au secteur ovin au titre de l'article 68 et qui ont nécessité le découplage total préalable de la prime à la brebis précédente le sont à hauteur de 22,5 euros par brebis, avec un minimum de 50 brebis par exploitation. Cette aide est conditionnée à une productivité d'au moins 0,5 agneau par brebis.

- Les 10 millions d'euros d'aides attribués au secteur caprin le sont à raison de 12,7 euros par chèvre, avec un seuil minimal de 25 têtes et un plafond de 400 têtes par exploitation.

- Les 30 millions d'euros affectés aux superficies de maïs fourrage le sont à raison de 20 euros par hectare. La mesure est plafonnée à 15 ha par exploitation et à ceux qui ont au moins $10 \mathrm{UGB}$.

- Les 45 millions d'euros alloués aux exploitations laitières de haute montagne, de montagne et de piémont, sont accordés à raison d'un maximum de 20 euros par 1000 litres, dans la limite d'un plafond qui sera fixé en fin de campagne (de l'ordre de 100000 litres par exploitation, en tenant compte de la transparence des GAEC).

- Pour les 4,6 millions d'euros ciblés sur la production de veaux sous la mère agréés label rouge ou produits dans le respect du règlement bio, le montant de l'aide sera de 50 euros par tête.

- Pour les 50 millions d'euros alloués en faveur de l'agriculture biologique, c'est une aide variable selon les catégories de cultures qui sera versée, ceci pour les agriculteurs déjà engagés en agriculture biologique.

- Une revalorisation de $15 \%$ de l'ICHN est effectuée sur les 25 premiers hectares.

- Les aides aux protéines végétales (100 euros par hectare), au blé dur en zone traditionnelle (30 euros par hectare), et la MAE rotationnelle sont allouées en fonction des assolements de 2007.

\section{3 / Les principaux effets du bilan de santé de la PAC}

Les résultats des simulations mettent en évidence que les systèmes affectés par la réorientation des aides directes sont en particulier ceux reposant sur les grandes cultures, alors que les bénéficiaires sont essentiellement les systèmes ruminants basés sur l'herbe (Chatellier et al 2009). Il apparaît aussi que de nombreuses exploitations agricoles françaises ne sont pas concernées par le rééquilibrage des aides directes, à savoir les productions viticoles, maraîchères, arboricoles et hors-sol. Les mesures adoptées répon- dent donc globalement bien à l'objectif initial d'un rééquilibrage des soutiens au profit des productions animales et des élevages les moins soutenus.

\section{1 / Des impacts différenciés sur les exploitations pratiquant l'élevage d'herbivores}

Les exploitations françaises orientées vers les productions d'herbivores se présentent donc de façon inégale face à la réorientation des soutiens envisagée dans le cadre du bilan de santé de la PAC (Institut de l'Elevage 2009). Les principaux facteurs qui sont à l'origine des impacts différenciés mesurés sont le type de production, la localisation géographique (plaine/montagne), l'assolement (prairies/céréales) ou le système technique (niveau d'intensification, inscription dans le cahier des charges de l'agriculture biologique...). Outre le ciblage de nouvelles aides sur tel ou tel système productif, l'impact varie d'une exploitation à l'autre en fonction de la nature et de l'importance du recours aux deux principaux outils de redistribution que sont l'article 63, en grande partie redistribué sur l'herbe productive mais à partir de prélèvements mixtes (au secteur végétal et dans une moindre mesure au secteur animal), et l'article 68 très ciblé sur certaines productions donc avec des effets pouvant être marqués pour certains systèmes.

L'impact du bilan de santé de la PAC est très nettement négatif pour les exploitations spécialisées de grandes cultures qui perdent en moyenne $16 \%$ de leurs aides directes (soit - 6100 euros par exploitation). Bien qu'en baisse, le niveau de soutien global dans ce secteur reste toutefois parmi les plus élevés par UTA. Pour les élevages d'herbivores, les résultats de la simulation sont présentés d'abord pour les systèmes spécialisés, puis pour les systèmes diversifiés (c'està-dire ceux pour lesquels la production dominante représente moins des deux tiers de l'activité globale).

a) Les exploitations spécialisées en élevage d'herbivores

Pour les éleveurs spécialisés en production ovine, l'augmentation des aides directes est forte en moyenne nationale, en raison notamment de l'octroi d'une nouvelle prime couplée à la brebis. Elle est estimée à $51 \%$ pour les exploitations d'ovins viande (soit + 14200 euros par exploitation) et à $37 \%$ pour les exploitations d'ovins laitiers (soit +8200 euros par exploitation). Après application des mesures, les exploitations spécialisées en ovins viande bénéficient d'un montant élevé d'aides directes (41 800 euros). Près du tiers de celles-ci relève du deuxième pilier de la PAC qui soutient la mise en valeur de territoires à fortes contraintes dans lesquels est désormais concentrée une forte proportion de ces élevages. Cette hausse importante des soutiens répond à l'objectif de rééquilibrage de revenus des élevages ovins, très inférieurs à la moyenne générale et de parité des aides du premier pilier avec l'élevage de vaches allaitantes. De fait, il ramène le montant des aides directes du premier pilier par emploi agricole au même niveau pour les deux types d'exploitations (ovins viande et bovins viande), sachant qu'elles conduisent des cheptels

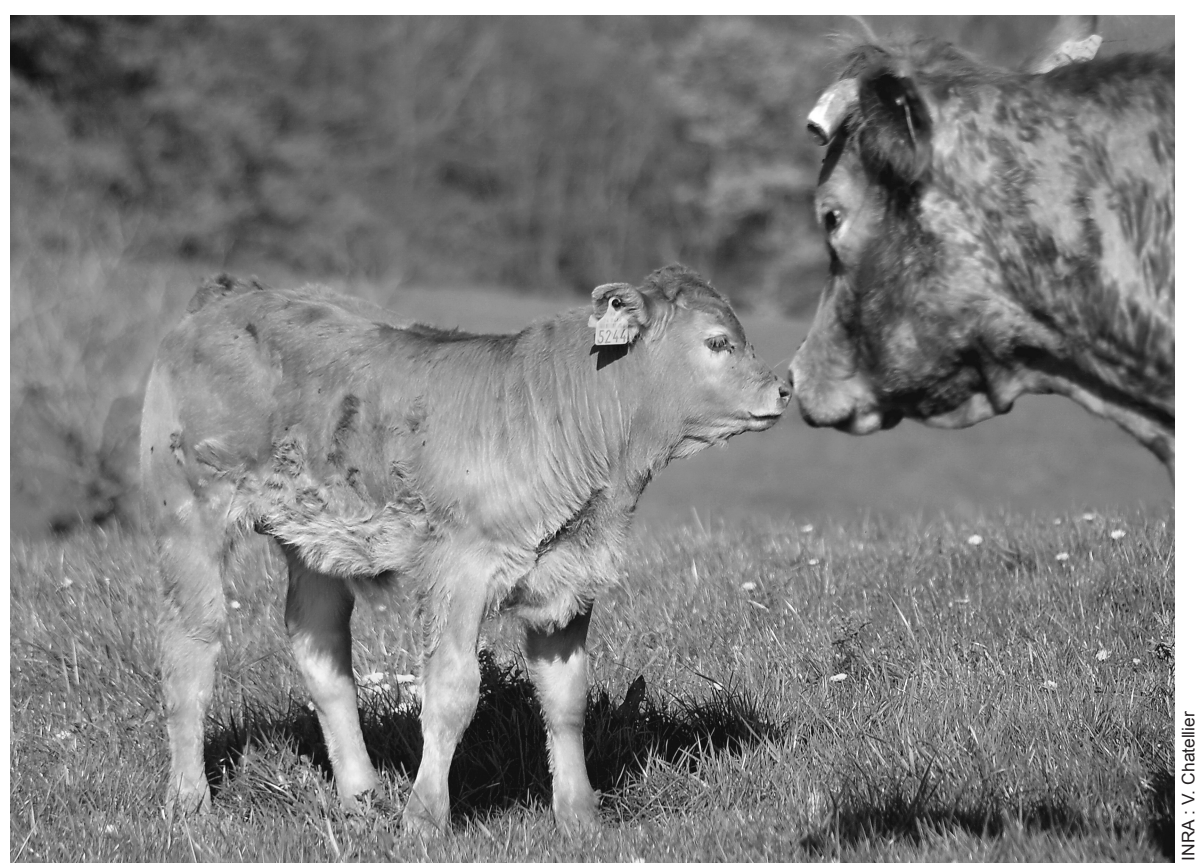

Un impact positif mais modeste pour le système naisseur 
Tableau 2. Caractéristiques moyennes des exploitations agricoles françaises en 2007 (d'après RICA 2007 - Traitement Institut de l'Elevage).

\begin{tabular}{|c|c|c|c|c|c|c|}
\hline & $\begin{array}{c}\text { Nom bre } \\
\text { exploitations }\end{array}$ & $\begin{array}{c}\text { UTA par } \\
\text { exploitation }\end{array}$ & $\begin{array}{c}\text { SAU par } \\
\text { exploitation } \\
\text { (hectares) }\end{array}$ & $\begin{array}{c}\text { Aides } \\
\text { directes par } \\
\text { exploitation } \\
\text { (euros) }\end{array}$ & $\begin{array}{c}\text { Aides } \\
\text { directes par } \\
\text { UTA } \\
\text { (euros) }\end{array}$ & $\begin{array}{c}\text { Aides } \\
\text { directes par } \\
\text { hectare de } \\
\text { SAU (euros) }\end{array}$ \\
\hline \multicolumn{7}{|c|}{ Exploitations spécialisées en élevage d'herbivores } \\
\hline Ovins-viande spécialisé & 6570 & 1,32 & 108 & 27600 & 20900 & 257 \\
\hline Ovins-lait & 5790 & 1,53 & 67 & 22400 & 14600 & 334 \\
\hline Caprins & 4560 & 2,13 & 66 & 17600 & 8300 & 267 \\
\hline Bovins-viande spécialisé & 38950 & 1,35 & 93 & 34900 & 25900 & 377 \\
\hline - dont Naisseur en montagne & 17920 & 1,25 & 87 & 33500 & 26700 & 384 \\
\hline - dont Naisseur - Herbager & 7880 & 1,42 & 109 & 37500 & 26400 & 345 \\
\hline - dont N-Engraisseur - Herbager & 1220 & 1,78 & 153 & 58400 & 32800 & 382 \\
\hline - dont N-Engraisseur Ouest et Plaine & 2390 & 1,58 & 89 & 37400 & 23600 & 419 \\
\hline Bovins-lait spécialisé & 41340 & 1,71 & 68 & 23600 & 13800 & 346 \\
\hline - dont herbager de montagne & 10410 & 1,56 & 71 & 20900 & 13400 & 293 \\
\hline - dont herbe-maïs de piémont & 4620 & 1,91 & 70 & 26300 & 13800 & 377 \\
\hline - dont herbager de plaine & 2430 & 1,65 & 72 & 19500 & 11800 & 269 \\
\hline - dont herbe-maïs de plaine & 8920 & 1,65 & 69 & 21900 & 13200 & 318 \\
\hline - dont intensif de plaine & 14960 & 1,79 & 64 & 26200 & 14600 & 409 \\
\hline \multicolumn{7}{|c|}{ Exploitations diversifiées ayant des herbivores } \\
\hline Ovins-viande diversifié & 2630 & 1,53 & 113 & 35400 & 23100 & 314 \\
\hline Bovins-viande diversifié & 31310 & 1,64 & 106 & 37200 & 22600 & 352 \\
\hline Bovins-lait diversifié & 49400 & 2,03 & 112 & 39900 & 19700 & 355 \\
\hline Engraisseur de bovins & 3250 & 1,70 & 89 & 39800 & 23400 & 449 \\
\hline \multicolumn{7}{|l|}{ Autres exploitations agricoles } \\
\hline Céréales & 60070 & 1,64 & 109 & 38900 & 23700 & 358 \\
\hline Autres (horticulture, viticulture...) & 82120 & 2,94 & 28 & 5100 & 1700 & 187 \\
\hline Toutes exploitations agricoles & 326010 & 2,00 & 80 & 27100 & 13600 & 339 \\
\hline
\end{tabular}

reproducteurs moyens de taille équivalente (450 brebis pour 60 vaches). Le rééquilibrage est très net pour les exploitations d'ovins allaitants à orientations «fourragères» (>1,4 UGB/ha), «herbagères» $(<1,4 \mathrm{UGB} / \mathrm{ha})$ et «pastorales» (qui dépassent $0,5 \mathrm{UGB} / \mathrm{ha}$ ). Il l'est un peu moins pour les exploitations les plus extensives $(<0,5 \mathrm{UGB} / \mathrm{ha})$.

Pour les éleveurs spécialisés en production caprine, l'impact de la réforme est nettement plus modeste $(+16 \%$ d'aides directes ou +2800 euros par exploitation). Cela s'explique surtout par le fait que ces exploitations ont souvent des grandes cultures associées à l'activité animale (ce qui implique un prélèvement budgétaire au titre de l'article 63) et des superficies fourragères moins conséquentes qu'en production ovine (tableau 2). Au final, et malgré les aides liées à l'article 68, les exploitations caprines restent parmi celles qui reçoivent le moins d'aides directes par emploi agricole (9600 euros).

Pour les éleveurs spécialisés de bovins-viande, les effets de la réorientation des soutiens sont, en moyenne nationale, nettement plus faibles que dans le secteur ovin $(+4 \%$ d'aides directes, soit +1200 euros par exploita- tion en moyenne). Si ce secteur est largement bénéficiaire des dotations de l'article 63 en raison de l'importance des surfaces en herbe, il contribue également aux prélèvements au travers de la PMTVA, de la PAB et des aides couplées aux surfaces céréalières (importantes chez les naisseurs-engraisseurs). D'une manière générale, le système «naisseur» est légèrement gagnant (environ $+5 \%$ d'aides directes) alors que la situation est inverse pour le système «naisseur-engraisseur» $(-2 \%$ en zone herbagère et $-4 \%$ dans l'ouest et en plaine). Pour mieux comprendre les effets croisés des prélèvements et des attributions, quelques précisions peuvent être apportées. Ainsi, pour percevoir en moyenne 4800 euros par exploitation via les allocations issues de l'article 63, les naisseurs-engraisseurs de l'Ouest subissent, en amont, un prélèvement de 3000 euros (le solde net est donc de 1800 euros, soit $39 \%$ de la dotation brute). Bien qu'atténué, le phénomène est le même pour les naisseursengraisseurs herbagers et les naisseurs qui ne perçoivent réellement que 52 et $66 \%$ des dotations brutes de l'article 63 Autrement dit, le nouveau soutien aux prairies qui compose l'essentiel de ces dotations est loin de représenter 80 euros net par hectare pour les éleveurs en bovins allaitants : le solde se rapproche plutôt de 37 euros par hectare pour un naisseur herbager et de 27 euros par hectare pour un naisseur-engraisseur de l'Ouest. La faiblesse de la variation nette des soutiens dans le secteur bovin allaitant spécialisé résulte aussi des niveaux initiaux de soutiens ; ce secteur contribue fortement aux prélèvements non sélectifs (article 68 et modulation) et n'est pas ciblé par les dotations visant un rééquilibrage spécifique des soutiens. Cependant, après application des mesures de redistribution, les systèmes bovins allaitants spécialisés conservent un niveau de soutien par emploi agricole familial parmi les plus élevés.

Pour les éleveurs bovins laitiers spécialisés, l'impact moyen national est modeste $(+1600$ euros d'aides directes, soit $+7 \%)$, mais il masque d'importants écarts selon les systèmes techniques et la localisation géographique (tableau 3). Les exploitations laitières spécialisées sont épargnées, pour une grande part, des prélèvements budgétaires opérés au titre de l'article 63, à l'exception des systèmes intensifs de plaine, qui ont des superficies importantes en maïs fourrage et céréales. Dotés de montants initiaux d'aides directes plus faibles que les exploitations spécialisées en 
Tableau 3. L'impact du bilan de santé de la PAC sur les exploitations agricoles françaises (d'après RICA 2007 - Traitement Institut de l'Elevage).

\begin{tabular}{|c|c|c|c|c|c|c|c|}
\hline & \multirow[b]{2}{*}{$\begin{array}{c}\text { Nombre } \\
\text { exploita- } \\
\text { tions }\end{array}$} & \multirow{2}{*}{$\begin{array}{c}\text { Impact } \\
\text { en } \\
\text { euros/ } \\
\text { exploitation }\end{array}$} & \multirow{2}{*}{$\begin{array}{c}\text { Impact } \\
\text { en } \% \\
\text { des } \\
\text { aides } \\
\text { directes } \\
\text { de } 2007\end{array}$} & \multicolumn{4}{|c|}{$\begin{array}{l}\text { Montant des aides directes } \\
\text { (après simulation des mesures } \\
\text { du bilan de santé) }\end{array}$} \\
\hline & & & & $\begin{array}{c}\text { Aides } \\
\text { directes } / \\
\text { exploitation }\end{array}$ & $\begin{array}{l}\text { Aides } \\
\text { directes } \\
\text { IUTA }\end{array}$ & $\begin{array}{c}\text { Aides } \\
\text { directes } \\
\text { /hectare } \\
\text { de SAU } \\
\text { (euros) }\end{array}$ & $\begin{array}{l}\text { Aides } \\
\text { directes } \\
\text { /Revenu } \\
(\%)\end{array}$ \\
\hline \multicolumn{8}{|c|}{ Exploitations spécialisées en élevage d'herbivores } \\
\hline Ovins-viande spécialisé & 6570 & 14200 & 51 & 41800 & 31600 & 389 & 339 \\
\hline Ovins-lait & 5790 & 8200 & 37 & 30600 & 19900 & 456 & 127 \\
\hline Caprins & 4560 & 2800 & 16 & 20400 & 9600 & 310 & 62 \\
\hline Bovins-viande spécialisé & 38950 & 1200 & 4 & 36100 & 26800 & 391 & 150 \\
\hline - dont Naisseur en montagne & 17920 & 1500 & 5 & 35000 & 27900 & 401 & 167 \\
\hline - dont Naisseur - Herbager & 7880 & 1500 & 4 & 39000 & 27400 & 359 & 144 \\
\hline $\begin{array}{l}\text { - dont N-Engraisseur - Herbager } \\
\text { - dont N-Engraisseur }\end{array}$ & 1220 & -1100 & -2 & 57400 & 32300 & 375 & 144 \\
\hline Ouest et Plaine & 2390 & -1400 & -4 & 36000 & 22700 & 404 & 93 \\
\hline Bovins-lait spécialisé & 41340 & 1600 & 7 & 25200 & 14700 & 370 & 81 \\
\hline - dont herbager de montagne & 10410 & 5700 & 27 & 26600 & 17100 & 373 & 122 \\
\hline - dont herbe-maïs de piémont & 4620 & 4800 & 18 & 31100 & 16300 & 446 & 117 \\
\hline - dont herbager de plaine & 2430 & 3200 & 17 & 22700 & 13800 & 314 & 66 \\
\hline - dont herbe-maïs de plaine & 8920 & 400 & 2 & 22200 & 13500 & 323 & 66 \\
\hline - dont intensif de plaine & 14960 & -1700 & -6 & 24500 & 13700 & 383 & 64 \\
\hline \multicolumn{8}{|c|}{ Exploitations diversifiées ayant des herbivores } \\
\hline Ovins-viande diversifié & 2630 & 4200 & 12 & 39600 & 25800 & 352 & 153 \\
\hline Bovins-viande diversifié & 31310 & -1400 & -4 & 35800 & 21800 & 339 & 113 \\
\hline Bovins-lait diversifié & 49400 & -1500 & -4 & 38500 & 19000 & 342 & 82 \\
\hline Engraisseur de bovins & 3250 & -4300 & -11 & 35400 & 20900 & 400 & 84 \\
\hline \multicolumn{8}{|l|}{ Autres exploitations agricoles } \\
\hline Céréales & 60070 & -6100 & -16 & 32800 & 20000 & 301 & 66 \\
\hline Autres (horticulture, viticulture...) & 82120 & -500 & -10 & 4600 & 1600 & 169 & 12 \\
\hline Toutes exploitations agricoles & 326010 & -800 & -3 & 26300 & 13200 & 330 & 70 \\
\hline
\end{tabular}

bovins-viande, elles subissent, en outre, de moindres prélèvements au titre de l'article 68 et de la modulation additionnelle. Les nouvelles aides directes allouées en faveur des superficies de prairies et de la production laitière de montagne bénéficient, en outre, à certaines d'entre elles. Ainsi, l'augmentation des aides directes est estimée à $27 \%$ pour les systèmes herbagers de montagne, $18 \%$ pour les systèmes mixtes «herbe-maïs» de piémont et $17 \%$ pour les systèmes herbagers de plaine (parfois plus dans le cas où ils relèvent de l'agriculture biologique). L'impact est, en revanche, neutre pour les systèmes mixtes «herbe-maïs» de plaine et légèrement négatif $(-6 \%)$ pour les systèmes intensifs. Cette réévaluation des soutiens tend cependant plus à combler le différentiel de revenu par emploi constaté par le passé qu'à anticiper les effets défavorables de la probable disparition des quotas laitiers sur les exploitations de montagne, moins flexibles, comme l'a montré le déroulement de la campagne 2007-2008 (avec des écarts de revenus qui ont atteint 10000 euros par UTA en défaveur de la montagne). b) Les exploitations diversifiées ayant des herbivores

Dans de nombreuses exploitations d'herbivores, le pôle animal n'est qu'une des composantes de l'activité économique globale. C'est souvent le cas dans les zones de plaine où l'essor des formes sociétaires contribue à ce que les unités diversifiées deviennent plus nombreuses. Dans la mesure où les orientations du bilan de santé de la PAC sont peu favorables aux productions végétales, les exploitations de polyculture-élevage sont moins favorisées que les unités spécialisées. Le montant des soutiens qui est attribué d'un côté (pour l'élevage ovin ou les superficies de prairies) est, en effet, prélevé de l'autre (cultures).

Pour les exploitations diversifiées ayant un atelier ovin-viande, la revalorisation des aides directes est estimée à $12 \%$ (soit +4200 euros par exploitation). L'impact est négatif pour les exploitations laitières diversifiées $(-4 \%)$ et les exploitations diversifiées de bovins-viande $(-4 \%)$. Au-delà de l'impact global par exploitation, les transferts internes d'aides viennent réévaluer la rentabilité de l'atelier d'élevage au sein de unités diversifiées en le rendant porteur d'une plus grande part des soutiens. Face aux tentations de mise en culture des prairies et de reconversion des surfaces fourragères labourables, cela peut favoriser le maintien des activités d'élevage dans ces structures. Dans les exploitations mixtes, l'atelier animal est, bien entendu, d'autant plus fragile que le prix des céréales est élevé relativement au prix des produits animaux comme cela fut le cas en 2007-2008.

La réorientation des aides directes évoquée ci-dessus devrait contribuer à rééquilibrer les revenus entre les différentes orientations de production (figure 1). Ce dernier restera cependant sous l'influence d'autres variables qui agissent parallèlement telles que les gains de productivité des facteurs de production et du travail, l'évolution de l'efficacité économique (économie d'échelle) et la dynamique des prix tant des produits agricoles que des différentes catégories d'intrants. 
Figure 1. Le revenu par UTA familiale dans les exploitations agricoles françaises avant et après le bilan de santé de la PAC (d'après RICA 2006-2007 - Traitement Institut de l'Elevage).

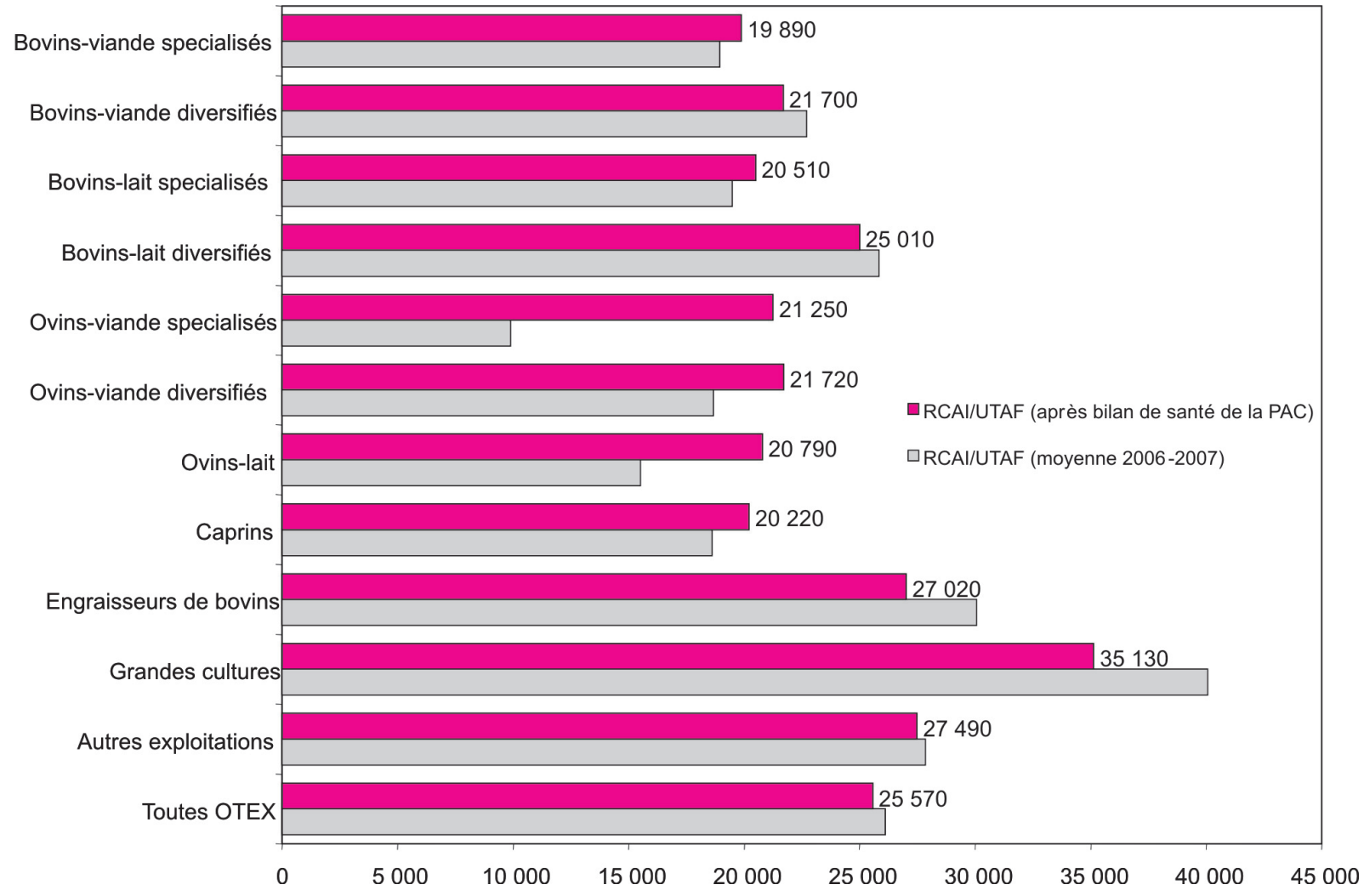

\section{2 / Une nette réorientation des aides en faveur des zones herba- gères et de montagne}

Le rééquilibrage des aides directes entre productions conduit à une réorientation des soutiens entre zones géographiques. Sans compter les sommes qui ne sont pas affectées dans les simulations conduites ici (assurance récolte, aides en faveur des nouveaux défis ou fond sanitaire), ce sont au moins 270 millions d'euros an qui devraient passer des zones de plaine vers les zones de montagne (Chatellier et Guyomard, 2009b, Institut de l'Elevage 2009). En montagne, et toutes orientations de production confondues, le gain représente l'équivalent de 4100 euros par exploitation, soit 53 euros par hectare. Il est plus important dans les exploitations ovines $(+46 \%$ d'aides directes) que dans les exploitations laitières $(+24 \%)$ ou bovines allaitantes $(+5 \%)$. Les zones de plaine perdent, quant à elles, 2500 euros par exploitation, soit 34 euros par hectare. Il en est de même pour les zones défavorisées simples qui voient leurs aides baisser de $2 \%$.

La réorientation des soutiens entre les régions administratives françaises réserve peu de surprise en ce sens qu'elle s'explique par les spécialisations agricoles (cf. carte). Les régions extensives d'élevage sont les plus favorisées par les mesures issues du bilan de santé de la PAC (Chatellier et Guyomard 2009a). Avec ses surfaces étendues de prairies localisées en zone de montagne et sa forte spécialisation laitière, l'Auvergne enregistre une hausse de ses soutiens de 65 millions d'euros, soit $+12 \%$. La situation est également favorable dans le Limousin $(+10 \%)$ où s'ajoutent aux surfaces d'herbe les productions de veaux sous la mère et de brebis. La grande région de Rhône-Alpes $(+8 \%)$ et la Franche-Comté $(+11 \%)$ bénéficient pleinement des soutiens à l'herbe et aux ateliers laitiers de montagne. Les régions ayant une part importante de céréales dans leur assolement sont, en revanche, perdantes. Les exploitations de l'Ile-de-France connaissent la baisse la plus importante $(-17 \%)$ devant les régions Centre, Champagne-Ardenne et Alsace (-13\%). La région intermédiaire du Poitou-Charentes participe à l'effort de prélèvement en perdant 32 millions d'euros de soutien (soit - 6\%), de même que les régions d'élevage intensif que sont les Pays de la Loire $(-4 \%)$ et la Bretagne $(-5 \%)$. La Bourgogne, région de polyculture-élevage et d'élevage allaitant perd 31 millions d'euros (- 5\%). En BasseNormandie, où les surfaces d'herbe sont équivalentes aux surfaces arables, le soutien ne devrait baisser que de $2 \%$. Les aides glissent toutefois d'Est en Ouest au sein de la région, de la zone de polyculture-élevage à la zone herba- gère. Même chose en Lorraine, où le volume d'aides baissera de $3 \%$ et où les soutiens migreront des zones de polyculture vers le Sud-Est herbager de la région. De même, en Midi-Pyrénées, la plaine céréalière de la Garonne cède une partie de son soutien aux contreforts Sud du Massif Central (ovins et herbe) et dans une moindre mesure à la zone pyrénéenne.

\section{Conclusion}

Les décisions françaises du bilan de santé de la PAC visent à mieux satisfaire plusieurs objectifs, dont une plus grande uniformisation des montants d'aides directes découplées rapportés à l'hectare, une allocation plus ciblée des fonds sur la protection des ressources naturelles et la rémunération de services non marchands (environnement et territoire), mais aussi un certain rééquilibrage des revenus (Ministère de l'Agriculture et de la Pêche 2009). Avec un montant annuel d'environ 1,3 milliard d'euros, la redistribution opérée est significative. Le rééquilibrage se fait principalement au détriment des producteurs de grandes cultures et au bénéfice des éleveurs d'herbivores, singulièrement ceux engagés dans des systèmes extensifs. En termes géographiques, la redistribution induira un transfert des régions localisées au nord d'une ligne Bordeaux-Strasbourg vers 
celles situées au sud de cette ligne, où se trouvent la quasi-totalité des zones défavorisées simples et des zones de montagne.

L'analyse des effets du bilan de santé de la PAC doit être replacée dans le contexte plus large des discussions, qui ont déjà commencé, sur les perspectives financières de l'Union européenne pour la période 2014 à 2020 . La question est de savoir si les décisions nationales suffiront pour justifier dans le long terme le «modèle agricole français» auprès des autres Etats membres, ceci dans un contexte où ils appliquent une politique agricole souvent plus simple, moins volontariste en termes d'équité de revenu, et où ils sont moins diversifiés en termes de productions agricoles, de structures et de territoires. Par ailleurs, la forte volatilité du prix des produits agricoles et des charges observée au cours des trois dernières années rappelle combien le revenu des différentes catégories d'agriculteurs est d'abord dépendant de la conjoncture économique. Si le rééquilibrage induit est ici potentiellement favorable aux élevages extensifs, une forte augmentation du prix des végétaux relativement au prix des produits animaux aurait un effet inverse qui annulerait le mouvement de rééquilibrage.
Dans cette perspective de renégociation, deux priorités pourraient être privilégiées : i) augmenter la part des fonds alloués en faveur de la gestion des risques (sanitaires, climatiques, de prix, de revenus); ii) accroître les budgets versés en faveur des «nouveaux défis» environnementaux (gestion quantitative et qualitative de l'eau, protection de la biodiversité, réduction des émissions de gaz à effet de serre). Plusieurs dispositions issues des décisions françaises, en premier lieu le maintien de la PHAE et la nouvelle aide aux surfaces d'herbe productives, contribuent à cette meilleure prise en compte des objectifs environnementaux et territoriaux. Elles seront en mesure de renforcer la légitimité du soutien public à l'agriculture française et à ses agriculteurs.

Suite à l'application des mesures, ne resteront couplées à la production que $75 \%$ de l'actuelle PMTVA (environ 600 millions d'euros par an), la nouvelle prime ovine et caprine (135 millions d'euros par an) et diverses primes de moindre importance soit au total 900 millions d'euros environ (alors que les aides couplées s'élevaient à 2,5 milliards d'euros en 2009). La France pourra objectivement défendre auprès de ses partenaires européens, le fait qu'elle a œuvré dans le sens d'une politique agricole davantage ciblée sur l'environnement et les territoires (via le nouveau soutien à l'herbe notamment). Cette nouvelle augmentation des soutiens découplés en proportion des soutiens totaux facilitera-t-elle la position communautaire dans la négociation sur le soutien interne à l'agriculture conduite dans le cadre de l'Organisation mondiale du commerce (OMC)? C'était aussi un objectif affiché par les négociateurs. Enfin, à l'objection que les aides découplées sont toujours octroyées sur la base du modèle historique, notre pays fera remarquer que le lien avec le passé de chaque exploitation a été significativement atténué. En d'autres termes, les choix récents vont aussi dans le sens d'une limitation assez significative des écarts dans le montant du paiement unique par hectare entre catégories d'agriculteurs.

La montée en puissance des soutiens découplés pose au moins deux questions qui devront susciter des analyses précises à soumettre aux décideurs politiques et aux responsables professionnels des filières : $i$ ) compte tenu de la forte volatilité des prix, pourra-t-on maintenir à long terme un système de soutien qui peut potentiellement

Carte. Impact du bilan de santé de la PAC sur l'évolution des aides directes dans les régions françaises (\% et millions d'euros) (d'après RICA 2007 - Traitement Institut de l'Elevage).

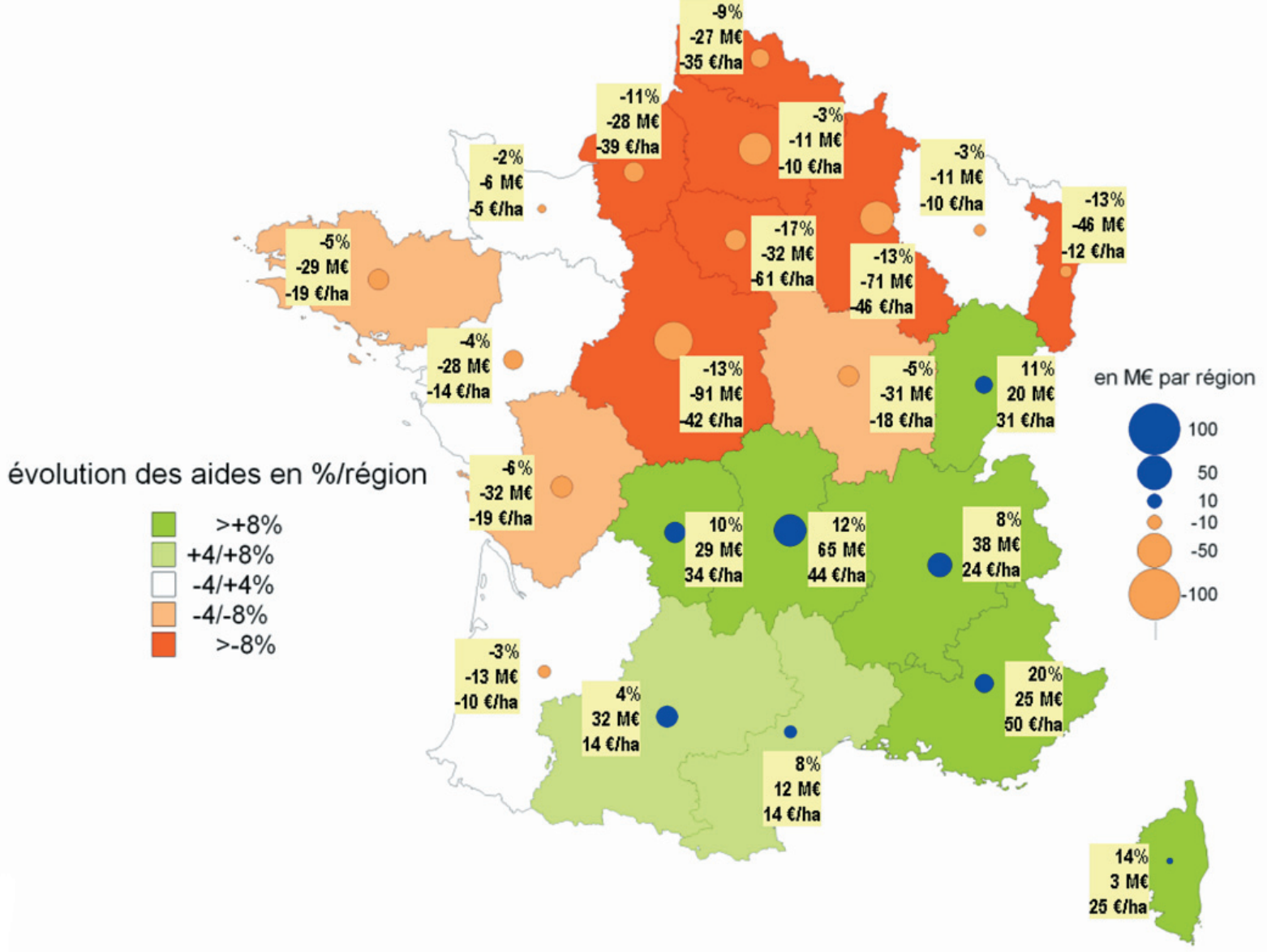


conduire à allouer des aides directes à des secteurs productifs bénéficiant d'une excellente conjoncture de prix pendant que d'autres secteurs font face à des difficultés de marché ? ii) quels seront les effets induits du découplage accentué sur la concentration géographique de l'offre et sur la dynamique des activités agricoles dans les zones défavorisées en termes de climat, relief ou potentiel agronomique (Chatellier et Guyomard 2008) sachant que la hausse des intrants depuis 2007 a réduit à néant l'incitation à produire exercée par les prix dans un grand nombre d'exploitations bovins ou ovins viande de ces zones (Institut de l'Elevage 2009). Si les décisions en France prises sont assez significatives quant à la réorientation des soutiens et à la prise en compte des enjeux environnementaux (Bureau et Mahé 2008), elles restent modestes sur la question des outils et des moyens budgétaires orientés vers la gestion des crises. Dans ce sens, l'actualité de la récente crise laitière vient souligner le vide laissé par les démantèlements des politiques antérieures de régulation et l'absence d'outils nouveaux adaptés à une certaine maîtrise de la volatilité des prix agricoles.

Le débat qui s'engage dans le cadre de la négociation budgétaire 2014-2020 s'ouvre à partir de positions très divergentes à la fois entre anciens et nouveaux Etats membres, mais aussi entre les Etats du noyau historique de l'Union européenne. L'interrogation quant à la légitimité d'une PAC devenue plus hétérogène, bien que financée sur fonds communautaires, risque d'être relancée et les appétits ne manqueront pas pour demander une affectation d'une partie des ressources agricoles sur des cibles que d'aucuns considéreront comme plus prioritaires, plus stratégiques, sources de croissance et de gains de productivité : formation, recherche, emploi, etc. Ceci d'autant plus qu'à l'interrogation quant aux objectifs de la PAC s'ajoutent la contrainte budgétaire globale et pour certains la perception d'une répartition inégalitaire et peu équitable de ces soutiens. La crise économique générale, les moyens engagés dans les plans de relance, dans l'innovation ou dans les mesures sociales, seront autant de raisons avancées et susceptibles de rendre difficile le maintien du budget accordé à l'agriculture. Cette application «à la française» de la dernière réforme peut semble-t-il donner quelques arguments au maintien d'une politique agricole et de ses moyens.

\section{Références}

APCA, 2009. La PAC, les décisions du bilan de santé. Chambres d'agriculture, 985, 11-50.

Bazin G., 2010. Quelle PAC après 2013 ? Comm. Acad. Agric. France, Paris, France, 12 p.

Boinon J.P., Kroll J.C., Lépicier D., Leseigneur A., Viallon J.B., 2008. La mise en oeuvre des DPU dans les États membres de l'Union européenne. Notes et Etudes Economiques, 31, 7-32.

Borzeix V., Codron S., Laureau D., Seban S. 2006. Pourquoi une nouvelle réforme de la Politique Agricole Commune? Notes et Etudes Economiques, 25, 7-44.

Boussard J.M., Trouvé A., 2010. For a new european agriculture and food policy that meets the challenges of this century. Report, 41p.

Bureau J.C., 2007. La PAC. Editions La découverte, Collection repères, Paris, France, $121 \mathrm{p}$.

Bureau J.C., Mahé L.P., 2008. La réforme de la PAC au-delà de 2013 : une vision à plus long terme. Rapport du Think tank «Notre Europe», 114p.

Bureau J.C., Witzke H.P, 2010. The single payment scheme after 2013: new approach, new targets. Report for Directorate General for internal policies (policy department B), 167p.

Butault J.P., Gohin A., Guyomard H., Barkaoui A., 2005. Une analyse économique de la réforme de la PAC de juin 2003. Revue Française d'Economie, 20, 57-108.

CCAN, 2010. Les concours publics à l'agriculture en 2009. Note pour la Commission des Comptes de l'Agriculture de la Nation, 20p.

Chatellier V., 2006. Le découplage et les droits à paiement unique dans les exploitations laitières et bovins viande. Cahiers d'Economie et Sociologie Rurales, 78, 53-80.

Chatellier V., Delattre F., 2005. Les soutiens directs et le découplage dans les exploitations agricoles de montagne. Economie rurale, 228, 40-57.

Chatellier V., Guyomard H., 2008. Le bilan de santé de la PAC, le découplage et l'élevage en zones difficiles. INRA Sci. Soc., 6, 8p.

Chatellier V., Guyomard H., 2009a. Le bilan de santé de la PAC et son application en France. Simulations et réflexions sur les décisions du 23 février 2009 du Ministre français de l'agriculture et de la pêche. Rapport de l'INRA (programme PSDR Grand-Ouest), $68 \mathrm{p}$.

Chatellier V., Guyomard H., 2009b. Le bilan de santé de la PAC : un effet positif pour les exploitations françaises de montagne. Bull. GTV, 49, 106-114.

Chatellier V., Guesdon J.C., Guyomard H., Perrot C., 2009. L'application française du bilan de santé de la PAC : un rééquilibrage favorable aux productions animales extensives. Renc. Rech. Rum., 203-210.

Conseil européen, 2009. Règlement (CE) n73/2009 du Conseil du 19 janvier 2009 établissant des règles communes pour les régimes de soutien direct en faveur des agriculteurs dans le cadre de la politique agricole commune, 243p.

Gohin A., 2009. Le bilan de santé de la Politique Agricole Commune : faut-il des exceptions au traitement général ? Economie et Prévision, 189, 1-20.

Guyomard H., Chatellier V., Courleux F., Levert F., 2007. La politique de soutien des revenus dans l'UE : quel avenir pour les droits à paiement unique ? Rapport du Conseil d'Analyse Economique (CAE), octobre, Paris, France, 125-179.

Institut de l'Elevage, 2009. Le bilan de santé de la PAC en France : un rééquilibrage en faveur de l'élevage. Dossier Economie de l'Elevage (hors-série), 41p.

Ministère de l'Agriculture et de la Pêche, 2009. Bilan de santé de la PAC : pour une PAC préventive, juste et durable, $4 \mathrm{p}$.

OCDE, 2001. Decoupling: A conceptual overview. OECD Paper, 10, 60p.

Piet L., Courleux F., Guyomard H., 2006. Les DPU : application en France et premiers éléments d'analyse économique. Notes et études économiques, $25,45-78$.

\section{Résumé}

Cet article présente une analyse des conséquences, pour les exploitations agricoles françaises, des décisions arrêtées dans le cadre du bilan de santé de la PAC par le Ministre de l'Agriculture et de la Pêche. Les simulations sont conduites, moyennant certaines hypothèses, à partir des données individuelles du Réseau d'Information Comptable Agricole (RICA). Elles permettent d'évaluer les impacts économiques des mesures adoptées pour différentes catégories d'exploitations agricoles réparties selon les systèmes de production et les régions. D'après ces travaux, la réorientation des aides directes sera favorable aux exploitations d'herbivores, principalement celles ayant une part élevée de prairies dans la surface agricole. Elle sera, en revanche, pénalisante pour les exploitations de grandes cultures et les élevages intensifs de bovins. La redistribution des aides directes induit un transfert des régions localisées au nord de la Loire vers celles situées en zones plus herbagères ou de montagne. La réorientation des soutiens entre les secteurs productifs, modeste mais hautement significative, vise à renforcer leur légitimité en les liant plus nettement à la rémunération de services environnementaux et territoriaux. 
Abstract

The extensive dairy farmers and sheep producers benefit from the French measures of the Health Check of the CAP

This paper presents an analysis of the consequences, for French farms, of the decisions made under the CAP Health Check by the Minister of Agriculture and Fisheries. Simulations were carried out thanks to the Farm Accountancy Data Network (FADN) and Network Livestock of the Institut de l'Elevage. They can, under certain assumptions of implementation, assess the economic impact of measures adopted in February 2009 (in terms of variation in farm income and direct payments) and discuss their effects on the dynamics of herbivorous productions. According to this work, the reorientation of supports will be favourable for grazing livestock farms, mainly those with a high proportion of grassland in agricultural land. It will, however, be economically disadvantageous for farm crops and intensively reared cattle. The redistribution of direct aid induced a transfer from regions located in the north of the Loire to those located in the south (especially in the mountains and areas with high proportions of grassland). The shift in support among the productive sectors, which was modest but highly significant, was aimed at enhancing their legitimacy by linking them more clearly to the remuneration of environmental and territorial services.

CHATELLIER V., GUESDON J.-C., GUYOMARD H., PERROT C., 2010. Les producteurs d'ovins et les éleveurs laitiers extensifs sont les principaux bénéficiaires de l'application française du bilan de santé de la PAC. Inra Prod. Anim., 23, $243-254$. 
